hep-ph/0309240

\title{
The electromagnetic vertex of neutrinos in an electron background and a magnetic field
}

\author{
José F. Nieves \\ Laboratory of Theoretical Physics, Department of Physics, P.O. Box 23343 \\ University of Puerto Rico, Rio Piedras, Puerto Rico 00931-3343
}

(Dated: September 2003)

\begin{abstract}
We study the electromagnetic vertex function of a neutrino that propagates in an electron background in the presence of a static magnetic field. The structure of the vertex function under the stated conditions is determined and it is written down in terms of a minimal and complete set of tensors. The one-loop expressions for all the form factors is given, up to terms that are linear in the magnetic field, and the approximate integral formulas that hold in the long wavelength limit are obtained. We discuss the physical interpretation of some of the form factors and their relation with the concept of the neutrino induced charge. The neutrino acquires a longitudinal and a transverse charge, due to the fact that the form factors depend on the transverse and longitudinal components of the photon momentum independently. We compute those form factors explicitly in various limiting cases and find that the longitudinal and transverse charge are the same for the case of a non-relativistic electron gas, but not otherwise.
\end{abstract}

\section{INTRODUCTION AND SUMMARY}

In the study of the electromagnetic properties of neutrinos in a medium, the two quantities of particular interest are the neutrino self-energy in the presence of an external magnetic field, and the neutrino electromagnetic vertex function. The former quantity determines the neutrino dispersion relation as it propagates in a magnetized medium, while the latter determines the relevant couplings in the calculation of the neutrino electromagnetic processes that occur in the medium, such as plasmon decay and Cherenkov radiation, among others. The electromagnetic couplings of the neutrino can be viewed in terms of effective dipole moments and an electric charge induced by the medium 1]. Although such identifications reveal some of the properties of the neutrino, both the intrinsic ones as well as those that result from its interaction with matter, for the practical calculation of the transition rates of the various processes the full electromagnetic vertex function is needed. Since the original calculations of those quantities more than a decade ago 2, 3, 4], some of their possible physical effects have been considered over the last few years [5, 6, 7, 8, 9, 10, 11, 12, 13, 14, 15. In addition, the calculations have been improved and refined using a variety of methods and techniques [16, 17, 18, 19].

Recently, there has been interest on another closely related quantity, namely the electromagnetic couplings of the neutrino when it propagates in a medium in the presence of a magnetic field. The main issue is here is to determine how the neutrino electromagnetic processes that take place in a medium, but in the absence of a magnetic field, are modified when a magnetic field is present. From a technical point of view, the relevant quantity is the neutrino electromagnetic vertex function, which must be calculated taking the presence of the magnetic field into account.

This problem has been approached from two points of view. On one hand, the vertex function has been calculated taking the magnetic field into account, but neglecting the effects of the matter medium [20]. On the other hand, the effect of both the magnetic field and the medium have been taken into account 21], but only for calculating the coupling that was identified as the induced neutrino charge, and not for the calculation of the full vertex function. Thus there does not exist a complete calculation of the full vertex function that takes into account the simultaneous presence of the magnetic field and the background medium.

In the this work we have taken precisely this problem. Our goal is to calculate the complete one-loop electromagnetic vertex function, including the effects of the medium and the linear terms in the magnetic field. To this end, we determine the most general form of the neutrino vertex function in the physical environment and under the conditions that we are considering. The vertex function is written in terms of a complete and minimal set of tensors, that are consistent with the requirements that follow from the chiral nature of the neutrino interactions and electromagnetic gauge invariance. Such a decomposition is generally useful in its own right, and it is also a useful reference point for the actual computation of the vertex function. Thus, we give the formulas for all the corresponding form factors in terms of momentum integrals over the background electron distribution functions. For arbitrary values of the photon momentum and/or general distributions functions the integrals are normally not doable, but they can be evaluated for various limiting cases and approximations that correspond to realistic situations. A particularly useful one that we consider is the so-called long wavelength limit, which is valid when the photon energy and momentum are smaller than the typical energy of an electron in the gas. In this limit the integrals that appear in the formulas for the form factors simplify but yet they remain valid for general forms of the momentum distributions, and for a wide range 
of values of the photon energy and momentum subject only to the restriction stated above. The simplified integral expressions so obtained can be used to compute explicitly the form factors for different kinematical regimes, such as the static limit (taking the photon energy to zero), and for various conditions of the background such as the classical or degenerate gas.

We observe that, apart from the photon energy, the form factors depend separately on the perpendicular and parallel components of the photon momentum. That is to say, if we denote those three quantities by $\omega, Q_{\perp}, Q_{\|}$respectively, the form factors depend on those three variables and not just on $\omega$ and $Q^{2}=Q_{\perp}^{2}+Q_{\|}^{2}$ as it is the case in the absence of the magnetic field. A consequence of this is that, in contrast to the latter case, there is no unique meaning to the concept of the induced neutrino charge, or in fact any other electromagnetic moment, when the magnetic field is non-zero. Briefly, the reason is that the zero (photon) momentum limit of the static form factors (i.e., evaluated for $\omega=0$ ) can be taken in two different ways, according to whether we set $Q_{\perp}=0$ first and $Q_{\|} \rightarrow 0$ afterwards or the other way around. If we insist on identifying a quantity such as the induced charge by looking at the static vertex function in the zero (photon) momentum limit, we are thus forced to define two different quantities, which in the specific case of the neutrino induced charge we denote by $e_{\nu}^{\|}$and $e_{\nu}^{\perp}$. We stress that the form factors have a different value in the two limits, and this is not a mathematical ambiguity but actually a reflection of the fact that, in the presence of the magnetic field, the two ways of taking the zero momentum limit correspond to different physical situations. As an illustration, and to further clarify some of these issues, we compute explicitly the form factors that are related to $e_{\nu}^{\|}$and $e_{\nu}^{\perp}$. As we show, $e_{\nu}^{\|}$and $e_{\nu}^{\perp}$ have the same value for the case of a non-relativistic electron gas, but they are given by different formulas otherwise. In order to establish contact with previous calculations [21], the

quantity calculated there is identified with what we call $e_{\nu}^{\|}$. However, some differences in the results are found and are noted.

In what follows we present the details of the calculations, divided as follows. An essential ingredient is the linearized form of the thermal propagator for an electron in a magnetic field. In Section 1 the linearized form is obtained from the Schwinger formula for the electron propagator, generalized to include the effects of the background medium, and approximating it up to terms that are linear in the magnetic field. In Section III the linearized propagator is used to obtain the one-loop formula for the neutrino electromagnetic vertex up to terms that are linear in the magnetic field. In Section IV we determine the structure of the vertex function and decompose it in terms of a minimal set of tensors. The end result of this procedure is a set of formulas for the corresponding form factors, expressed as integrals over the momentum distribution functions of the electrons in the medium. In Section $\nabla$ the approximate formulas that are valid in the long wavelength limit are derived first, and they are then used to evaluate explicitly the form factors that are related to $e_{\nu}^{\|}$and $e_{\nu}^{\perp}$ for various specific cases. Section VI contains our conclusions. In addition there are two appendices. The general decomposition of the vertex function is guided by several requirements, such as the transversality condition which of course is a general consequence of the electromagnetic gauge invariance. However, it is neither obvious to see nor trivial to prove, that the one-loop expression for the vertex function satisfies the transversality condition. The proof that it in fact does so is supplied in those appendices.

\section{THE LINEARIZED ELECTRON PROPAGATOR}

In the notation of Ref. [22], the Schwinger propagator for the electron in a magnetic field can be written in the form

$$
i S_{F}(p)=\int_{0}^{\infty} d \tau G(p, s) e^{i \tau \Phi(p, s)-\epsilon \tau}
$$

where the variable $s$ is defined by

$$
s=|e| B \tau
$$

and the exact formulas for $G(p, s)$ and $\Phi(p, s)$ are given in Eq. (3.4) of that reference. The linear approximation that we propose to use consists in retaining up to terms that are linear in $B$. To this order,

$$
\begin{aligned}
& \Phi=p^{2}-m^{2} \\
& G=\not p+m+i \tau|e| B G_{B},
\end{aligned}
$$

where

$$
G_{B}=\gamma_{5}[(p \cdot b) \psi-(p \cdot u) \not b+m \psi \not b],
$$


so that

$$
S_{F}=S_{0}+S_{B},
$$

where

$$
\begin{aligned}
i S_{0} & =i \frac{\not p+m}{p^{2}-m^{2}} \\
i S_{B} & =\frac{-i}{\left(p^{2}-m^{2}\right)^{2}}\left(|e| B G_{B}\right) .
\end{aligned}
$$

The vector $u^{\mu}$ that appears in Eq. (2.4) is the velocity four-vector of the medium which, in the frame of reference in which the medium is at rest, takes the form

$$
u^{\mu}=(1,0)
$$

We have also introduced the vector $b^{\mu}$ which is such that, in that frame,

$$
b^{\mu}=(0, \hat{b})
$$

where the magnetic field is given by

$$
\vec{B}=B \hat{b}
$$

The thermal electron propagator which, in addition to the magnetic field, incorporates the effect of the electron background, is given by

$$
S_{e}=S_{F}-\left[S_{F}-\bar{S}_{F}\right] \eta_{e}
$$

where

$$
\eta_{e}(p \cdot u)=\theta(p \cdot u) f_{e}(p \cdot u)+\theta(-p \cdot u) f_{\bar{e}}(-p \cdot u)
$$

with

$$
\begin{aligned}
& f_{e}(x)=\frac{1}{e^{\beta\left(x-\mu_{e}\right)}+1} \\
& f_{\bar{e}}(x)=\frac{1}{e^{\beta\left(x+\mu_{e}\right)}+1} .
\end{aligned}
$$

Here $\beta$ stands for the inverse temperature and $\mu_{e}$ the electron chemical potential. Using Eqs. (2.5), (2.6), (2.4), the complete propagator can be expressed as

$$
S_{e}=S_{0}+S_{B}+S_{T}+S_{T B}
$$

where

$$
\begin{aligned}
i S_{T} & =-2 \pi \delta\left(p^{2}-m^{2}\right) \eta_{e}(\not p+m) \\
i S_{T B} & =-2 \pi \delta^{\prime}\left(p^{2}-m^{2}\right) \eta_{e}\left(|e| B G_{B}\right),
\end{aligned}
$$

where $G_{B}$ is given in Eq. (2.4) and the notation $\delta^{\prime}$ denotes the derivative of the delta function with respect to its argument.

Although we will not give the details here, it is reassuring to mention that when this linearized propagator is used to compute the self-energy diagrams for the neutrino in an electron gas, the usual expression for the magnetic field contribution to the neutrino dispersion relation, or equivalently the index refraction, is reproduced.

\section{CALCULATION OF THE VERTEX FUNCTION}

The off-shell neutrino electromagnetic vertex function $\Gamma^{\mu}$ is defined such that the matrix element of the electromagnetic current between neutrino states with momenta $k$ and $k^{\prime}$ is given by

$$
\left\langle k^{\prime}\left|j_{\mu}(0)\right| k\right\rangle=\bar{u}\left(k^{\prime}\right) \Gamma_{\mu} u(k) .
$$


(a)

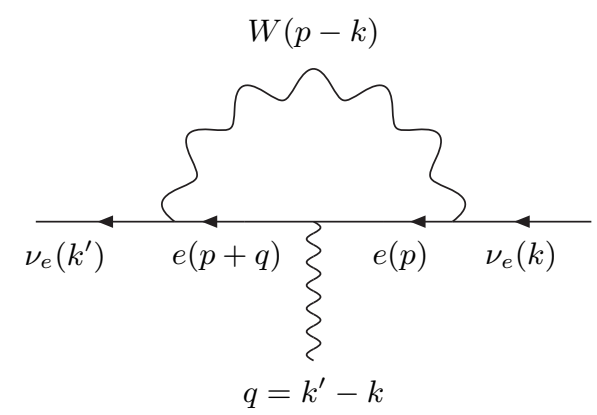

(b)

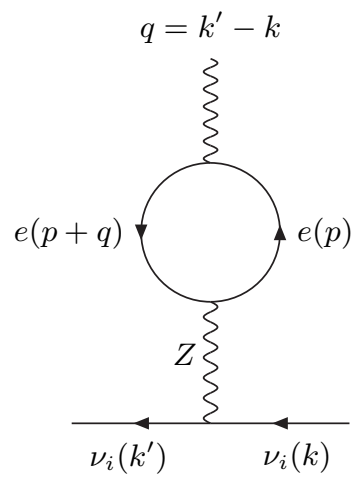

FIG. 1: One-loop diagrams for the neutrino electromagnetic vertex in an electron background, to order $1 / M_{W}^{2}$.

To order $1 / M_{W}^{2}$, the one-loop diagrams that are relevant to the calculation of $\Gamma^{\mu}$ are shown in Fig. 1 Their contributions are

$$
\begin{aligned}
-i \Gamma_{\nu}^{(W)}(q) & =-\left(\frac{e g^{2}}{2 M_{W}^{2}}\right) \int \frac{d^{4} p}{(2 \pi)^{4}} \gamma^{\mu} L i S_{e}(p+q) \gamma_{\nu} i S_{e}(p) \gamma_{\mu} L \\
-i \Gamma_{\nu}^{(Z)}(q) & =\left(\frac{e g^{2}}{4 \cos ^{2} \theta_{W} M_{Z}^{2}}\right) \gamma^{\mu} L \int \frac{d^{4} p}{(2 \pi)^{4}} \operatorname{Tr}\left[i S_{e}(p+q) \gamma_{\nu} i S_{e}(p) \gamma_{\mu}\left(a_{e}+b_{e} \gamma_{5}\right)\right],
\end{aligned}
$$

where

$$
q=k^{\prime}-k
$$

is the momentum of the incoming photon, $L=\frac{1}{2}\left(1-\gamma_{5}\right)$ and, in the standard model

$$
\begin{aligned}
& a_{e}=-\frac{1}{2}+2 \sin ^{2} \theta_{W}, \\
& b_{e}=\frac{1}{2} .
\end{aligned}
$$

Using the identity

$$
\gamma^{\mu} L M \gamma_{\mu} L=-\operatorname{Tr}\left(M \gamma_{\mu} L\right) \gamma^{\mu} L,
$$

which holds for any $4 \times 4$ matrix $M$, the charge current contribution can be put in the same form as the neutral current one, namely

$$
-i \Gamma_{\nu}^{(W)}(q)=\left(\frac{e g^{2}}{2 M_{W}^{2}}\right) \gamma^{\mu} L \int \frac{d^{4} p}{(2 \pi)^{4}} \operatorname{Tr}\left[i S_{e}(p+q) \gamma_{\nu} i S_{e}(p) \gamma_{\mu} L\right]
$$


When Eq. (2.13) is substituted in the above expressions for $\Gamma_{\nu}^{(W, Z)}$, several terms are generated and we want to retain only those that, schematically, involve the products $S_{T B} S_{0}$ or $S_{T} S_{B}$, i.e., the terms that depend both on $B$ and the temperature. Proceeding in this way, we then have

$$
\begin{aligned}
\Gamma_{\nu}^{(W)} & =-\left(\frac{e g^{2}}{4 M_{W}^{2}}\right) \gamma^{\mu} L\left(T_{\mu \nu}^{(V)}+T_{\mu \nu}^{(A)}\right) \\
\Gamma_{\nu}^{(Z)} & =-\left(\frac{e g^{2}}{4 M_{W}^{2}}\right) \gamma^{\mu} L\left(a_{e} T_{\mu \nu}^{(V)}-b_{e} T_{\mu \nu}^{(A)}\right)
\end{aligned}
$$

where

$$
\begin{aligned}
T_{\mu \nu}^{(V)}= & i \int \frac{d^{4} p}{(2 \pi)^{4}} \operatorname{Tr} \gamma_{\mu}\left[S_{0}\left(p^{\prime}\right) \gamma_{\nu} S_{T B}(p)+S_{T B}\left(p^{\prime}\right) \gamma_{\nu} S_{0}(p)\right. \\
& \left.+S_{T}\left(p^{\prime}\right) \gamma_{\nu} S_{B}(p)+S_{B}\left(p^{\prime}\right) \gamma_{\nu} S_{T}(p)\right], \\
T_{\mu \nu}^{(A)}= & i \int \frac{d^{4} p}{(2 \pi)^{4}} \operatorname{Tr} \gamma_{5} \gamma_{\mu}\left[S_{0}\left(p^{\prime}\right) \gamma_{\nu} S_{T B}(p)+S_{T B}\left(p^{\prime}\right) \gamma_{\nu} S_{0}(p)\right. \\
& \left.+S_{T}\left(p^{\prime}\right) \gamma_{\nu} S_{B}(p)+S_{B}\left(p^{\prime}\right) \gamma_{\nu} S_{T}(p)\right],
\end{aligned}
$$

and we have defined

$$
p^{\prime}=p+q .
$$

The vertex function for the electron neutrino is $\Gamma_{\nu}^{(W)}+\Gamma_{\nu}^{(Z)}$, while for the other neutrino flavors it is just $\Gamma_{\nu}^{(Z)}$. Denoting a given neutrino flavor by $\ell=e, \mu, \tau$, then the neutrino vertex function is

$$
\Gamma_{\nu}^{(\ell)}=-\left(\frac{e g^{2}}{4 M_{W}^{2}}\right) \gamma^{\mu} L\left(\chi_{V}^{(\ell)} T_{\mu \nu}^{(V)}+\chi_{A}^{(\ell)} T_{\mu \nu}^{(A)}\right)
$$

where

$$
\begin{aligned}
& \chi_{V}^{(\ell)}=\delta_{\ell, e}+a_{e}, \\
& \chi_{A}^{(\ell)}=\delta_{\ell, e}-b_{e} .
\end{aligned}
$$

All the four terms in Eq. 3.9 involve the traces

$$
\begin{aligned}
& 4 L_{\mu \nu}^{(1)}=\operatorname{Tr} \gamma_{\mu}\left(\not p^{\prime}+m\right) \gamma_{\nu} G_{B}(p) \\
& 4 L_{\mu \nu}^{(2)}=\operatorname{Tr} \gamma_{\mu} G_{B}\left(p^{\prime}\right) \gamma_{\nu}(\not p+m)
\end{aligned}
$$

and analogously in Eq. (3.10),

$$
\begin{aligned}
4 K_{\mu \nu}^{(1)} & =\operatorname{Tr} \gamma_{5} \gamma_{\mu}\left(\not p^{\prime}+m\right) \gamma_{\nu} G_{B}(p, \\
4 K_{\mu \nu}^{(2)} & =\operatorname{Tr} \gamma_{5} \gamma_{\mu} G_{B}\left(p^{\prime}\right) \gamma_{\nu}(\not p+m) .
\end{aligned}
$$

It is useful to observe that $L_{\mu \nu}^{(2)}=L_{\nu \mu}^{(1)}\left(p^{\prime} \leftrightarrow p\right)$ and $K_{\mu \nu}^{(2)}=K_{\mu \nu}^{(1)}\left(p \leftrightarrow p^{\prime}\right)$. Then, by standard Dirac matrix algebra we find

$$
\begin{aligned}
L_{\mu \nu}^{(1)} & =i \epsilon_{\mu \nu \alpha \beta}\left(p \cdot u p^{\alpha} b^{\beta}-p \cdot b p^{\alpha} u^{\beta}-m^{2} u^{\alpha} b^{\beta}\right) \\
L_{\mu \nu}^{(2)} & =i \epsilon_{\mu \nu \alpha \beta}\left(-p^{\prime} \cdot u p^{\alpha} b^{\beta}+p^{\prime} \cdot b p^{\alpha} u^{\beta}+m^{2} u^{\alpha} b^{\beta}\right) \\
K_{\mu \nu}^{(1)} & =p \cdot u\left(p_{\mu}^{\prime} b_{\nu}+b_{\mu} p_{\nu}^{\prime}-p^{\prime} \cdot b g_{\mu \nu}\right)-p \cdot b\left(p_{\mu}^{\prime} u_{\nu}+u_{\mu} p_{\nu}^{\prime}-p^{\prime} \cdot u g_{\mu \nu}\right)-m^{2}\left(u_{\mu} b_{\nu}-b_{\mu} u_{\nu}\right) \\
K_{\mu \nu}^{(2)} & =p^{\prime} \cdot u\left(p_{\mu} b_{\nu}+b_{\mu} p_{\nu}-p \cdot b g_{\mu \nu}\right)-p^{\prime} \cdot b\left(p_{\mu} u_{\nu}+u_{\mu} p_{\nu}-p \cdot u g_{\mu \nu}\right)-m^{2}\left(u_{\mu} b_{\nu}-b_{\mu} u_{\nu}\right) .
\end{aligned}
$$

Going back to Eqs. (3.9) and (3.10) and using the above formulas for the traces, we obtain

$$
\begin{aligned}
& T_{\mu \nu}^{(V)}=4|e| B \int \frac{d^{4} p}{(2 \pi)^{3}} \eta_{e}(p . u)\left\{\frac{-L_{\mu \nu}^{(1)} \delta^{\prime}\left(p^{2}-m^{2}\right)}{(p+q)^{2}-m^{2}}+\frac{L_{\mu \nu}^{(2)} \delta\left(p^{2}-m^{2}\right)}{\left[(p+q)^{2}-m^{2}\right]^{2}}-(q \rightarrow-q)\right\}, \\
& T_{\mu \nu}^{(A)}=4|e| B \int \frac{d^{4} p}{(2 \pi)^{3}} \eta_{e}(p . u)\left\{\frac{-K_{\mu \nu}^{(1)} \delta^{\prime}\left(p^{2}-m^{2}\right)}{(p+q)^{2}-m^{2}}+\frac{K_{\mu \nu}^{(2)} \delta\left(p^{2}-m^{2}\right)}{\left[(p+q)^{2}-m^{2}\right]^{2}}+(q \rightarrow-q)\right\} .
\end{aligned}
$$


In arriving at this expression we have used the standard trick of making the change of variables $p \rightarrow p-q$ in those terms where the factor $\eta_{e}\left(p^{\prime}\right)$ appears, together with the properties

$$
\begin{aligned}
L_{\mu \nu}^{(1)}(p \rightarrow p-q) & =-L_{\mu \nu}^{(2)}(q \rightarrow-q), \\
L_{\mu \nu}^{(2)}(p \rightarrow p-q) & =-L_{\mu \nu}^{(1)}(q \rightarrow-q), \\
K_{\mu \nu}^{(1)}(p \rightarrow p-q) & =K_{\mu \nu}^{(2)}(q \rightarrow-q), \\
K_{\mu \nu}^{(2)}(p \rightarrow p-q) & =K_{\mu \nu}^{(1)}(q \rightarrow-q),
\end{aligned}
$$

which can be seen by inspection of Eq. (3.16).

\section{STRUCTURE OF THE VERTEX FUNCTION}

In the context of the one loop calculation that we are considering, the conservation of the electron vector current implies that the tensors $T_{\mu \nu}^{(V, A)}$ satisfy the transversality relations

$$
\begin{aligned}
q^{\nu} T_{\mu \nu}^{(V)}=q^{\mu} T_{\mu \nu}^{(V)} & =0, \\
q^{\nu} T_{\mu \nu}^{(A)} & =0 .
\end{aligned}
$$

It is neither immediately evident nor trivial to prove that the one-loop formulas given in Eq. (3.17) satisfy such relations but, as we show explicitly in Appendices $\mathrm{A}$ and $\mathrm{B}$ they do. Similarly, there we also verify that, in contrast to the situation without the magnetic field, in the present case $q^{\mu} T_{\mu \nu}^{(A)} \neq 0$.

To exploit Eq. (4.1) we introduce the following set of mutually orthogonal vectors

$$
\begin{aligned}
& \tilde{u}_{\mu}=\tilde{g}_{\mu \nu} u^{\nu}, \\
& \tilde{b}_{\mu}=\tilde{g}_{\mu \nu} b^{\nu}-\frac{q \cdot u}{q^{2}}\left(q \cdot u b_{\mu}-q \cdot b u_{\mu}\right), \\
& \tilde{t}_{\mu}=\epsilon_{\mu \alpha \beta \gamma} u^{\alpha} b^{\beta} q^{\gamma},
\end{aligned}
$$

where

$$
\tilde{g}_{\mu \nu} \equiv g_{\mu \nu}-\frac{q_{\mu} q_{\nu}}{q^{2}} .
$$

These vectors are transverse to $q_{\mu}$ and satisfy the closure relation

$$
\frac{\tilde{u}_{\mu} \tilde{u}_{\nu}}{\tilde{u}^{2}}+\frac{\tilde{b}_{\mu} \tilde{b}_{\nu}}{\tilde{b}^{2}}+\frac{\tilde{t}_{\mu} \tilde{t}_{\nu}}{\tilde{t}^{2}}=\tilde{g}_{\mu \nu}
$$

It is useful to note that $\tilde{b}^{\mu}$ and $\tilde{t}^{\mu}$ also satisfy

$$
\tilde{b} \cdot u=\tilde{t} \cdot u=0
$$

\section{A. The structure of $T_{\mu \nu}^{(V)}$}

The transversality of $T_{\mu \nu}^{(V)}$ with respect to both indices implies that it can be expanded in terms of the nine bilinear products that can be formed out of the set of vectors $\{\tilde{u}, \tilde{b}, \tilde{t}\}$. Therefore, we define the following three diagonal tensors

$$
\begin{aligned}
Q_{\mu \nu} & =\frac{\tilde{u}_{\mu} \tilde{u}_{\nu}}{\tilde{u}^{2}}, \\
B_{\mu \nu} & =\frac{\tilde{b}_{\mu} \tilde{b}_{\nu}}{\tilde{b}^{2}}, \\
R_{\mu \nu} & =\tilde{g}_{\mu \nu}-Q_{\mu \nu}-B_{\mu \nu},
\end{aligned}
$$


the three symmetric combinations

$$
\begin{aligned}
& S_{1 \mu \nu}=\tilde{b}_{\mu} \tilde{t}_{\nu}+(\mu \leftrightarrow \nu), \\
& S_{2 \mu \nu}=\tilde{u}_{\mu} \tilde{t}_{\nu}+(\mu \leftrightarrow \nu), \\
& S_{3 \mu \nu}=\tilde{u}_{\mu} \tilde{b}_{\nu}+(\mu \leftrightarrow \nu),
\end{aligned}
$$

and the three antisymmetric ones

$$
\begin{aligned}
& A_{1 \mu \nu}=\tilde{b}_{\mu} \tilde{t}_{\nu}-(\mu \leftrightarrow \nu), \\
& A_{2 \mu \nu}=\tilde{u}_{\mu} \tilde{t}_{\nu}-(\mu \leftrightarrow \nu), \\
& A_{3 \mu \nu}=\tilde{u}_{\mu} \tilde{b}_{\nu}-(\mu \leftrightarrow \nu) .
\end{aligned}
$$

$A_{1 \mu \nu}$ and $A_{2 \mu \nu}$ can be expressed in a more convenient form by using the relations

$$
\begin{gathered}
\tilde{u}_{\nu} \epsilon_{\mu \alpha \beta \gamma} \tilde{u}^{\alpha} \tilde{b}^{\beta} q^{\gamma}-(\mu \leftrightarrow \nu)=\tilde{u}^{2} \epsilon_{\mu \nu \alpha \beta} \tilde{b}^{\alpha} q^{\beta} \\
\tilde{b}_{\nu} \epsilon_{\mu \alpha \beta \gamma} \tilde{b}^{\alpha} \tilde{u}^{\beta} q^{\gamma}-(\mu \leftrightarrow \nu)=\tilde{b}^{2} \epsilon_{\mu \nu \alpha \beta} \tilde{u}^{\alpha} q^{\beta}
\end{gathered}
$$

which follow from contracting the identity

$$
g_{\lambda \nu} \epsilon_{\mu \gamma \alpha \beta}-g_{\lambda \mu} \epsilon_{\nu \gamma \alpha \beta}+g_{\lambda \gamma} \epsilon_{\nu \mu \alpha \beta}-g_{\lambda \alpha} \epsilon_{\nu \mu \gamma \beta}+g_{\lambda \beta} \epsilon_{\nu \mu \gamma \alpha}=0
$$

with $\tilde{u}^{\lambda} \tilde{u}^{\nu} \tilde{b}^{\alpha} q^{\beta}$ in one case, and with $\tilde{b}^{\lambda} \tilde{b}^{\nu} \tilde{u}^{\alpha} q^{\beta}$ in the other. Therefore, instead of the antisymmetric combinations $A_{1,2}$, we will use instead

$$
\begin{aligned}
\tilde{P}_{1 \mu \nu} & \equiv i \epsilon_{\mu \nu \alpha \beta} \tilde{u}^{\alpha} q^{\beta}, \\
\tilde{P}_{2 \mu \nu} & \equiv i \epsilon_{\mu \nu \alpha \beta} \tilde{b}^{\alpha} q^{\beta} .
\end{aligned}
$$

Two additional properties of the one-loop formula for $T_{\mu \nu}^{(V)}$, which follow from inspection of Eq. (3.17), are: (i) it is antisymmetric, and (ii) it transforms as a pseudo-tensor under parity. Out of the set of nine tensors defined above, only $\tilde{P}_{1}$ and $\tilde{P}_{2}$ share both properties. It is then clear that, to this order, the structure of $T_{\mu \nu}^{(V)}$ is simply

$$
T_{\mu \nu}^{(V)}=\tilde{T}_{1}^{(V)} \tilde{P}_{1 \mu \nu}+\tilde{T}_{2}^{(V)} \tilde{P}_{2 \mu \nu}
$$

The form factors $T_{1,2}^{(V)}$ are, in general, functions of the three scalar variables $\omega, Q_{\|}$and $Q_{\perp}$ which are defined by

$$
\begin{aligned}
\omega & =q \cdot u, \\
Q_{\|} & =-q \cdot b, \\
Q_{\perp} & =\sqrt{Q^{2}-Q_{\|}^{2}},
\end{aligned}
$$

where

$$
Q^{2}=|\vec{Q}|^{2}=\omega^{2}-q^{2}
$$

These variables correspond to the photon energy in the rest frame of the medium and the components of the photon momentum $\vec{Q}$ that are parallel and perpendicular, respectively, to $\vec{B}$ in this frame. It is important to stress that in general, besides $\omega$, the form factors introduced in Eq. (4.12) depend on the variables $Q_{\perp}$ and $Q_{\|}$, separately. This contrasts with the situation without the magnetic field, in which case the form factors depend only on $\omega$ and $Q$. As we will see in Section $\nabla B$ this requires some extra care if we want to interpret the form factors in terms of the static electromagnetic moments of the neutrino.

The form factors are determined by contracting both sides of Eq. (3.17) with the tensors $\tilde{P}_{1,2}$ and using the relations

$$
\begin{aligned}
\tilde{P}_{1}^{2} & =-2 Q^{2}, \\
\tilde{P}_{2}^{2} & =-2 Q^{2} \frac{Q_{\perp}^{2}}{q^{2}}, \\
\tilde{P}_{1} \tilde{P}_{2} & =0,
\end{aligned}
$$


with $\tilde{P}_{i} \tilde{P}_{j}=\tilde{P}_{i}^{\mu \nu} \tilde{P}_{j \mu \nu}$. In this way, we obtain

$$
\begin{aligned}
& \tilde{T}_{1}^{(V)}=\frac{-1}{Q^{2}} T_{1}^{(V)}, \\
& \tilde{T}_{2}^{(V)}=\frac{1}{Q_{\perp}^{2}}\left(T_{2}^{(V)}+\frac{\omega Q_{\|}}{Q^{2}} T_{1}^{(V)}\right),
\end{aligned}
$$

where $T_{1,2}^{(V)}$ are the integrals

$$
T_{i}^{(V)}=4|e| B \int \frac{d^{4} p}{(2 \pi)^{3}} \eta_{e}(p . u)\left\{\frac{-L_{i}^{(1)} \delta^{\prime}\left(p^{2}-m^{2}\right)}{(p+q)^{2}-m^{2}}+\frac{L_{i}^{(2)} \delta\left(p^{2}-m^{2}\right)}{\left[(p+q)^{2}-m^{2}\right]^{2}}+(q \rightarrow-q)\right\},
$$

with

$$
\begin{aligned}
& L_{1}^{(1)}=\left(p_{u}+q_{u}\right) K+p_{b}\left(p \cdot q+q^{2}\right)-m^{2} q_{b}, \\
& L_{1}^{(2)}=-p_{u} K-\left(p_{b}+q_{b}\right) p \cdot q+m^{2} q_{b}, \\
& L_{2}^{(1)}=\left(p_{b}+q_{b}\right) K+p_{u}\left(p \cdot q+q^{2}\right)-m^{2} q_{u}, \\
& L_{2}^{(2)}=-p_{b} K-\left(p_{u}+q_{u}\right) p \cdot q+m^{2} q_{u} .
\end{aligned}
$$

In writing the last equation we have introduced the factor

$$
K=p_{u} q_{b}-p_{b} q_{u},
$$

and used the shorthand notation

$$
\begin{aligned}
& p_{u}=p \cdot u \\
& p_{b}=p \cdot b,
\end{aligned}
$$

and similarly for $q_{u}$ and $q_{b}$.

\section{B. The structure of $T_{\mu \nu}^{(A)}$}

Since $T_{\mu \nu}^{(A)}$ satisfies $q^{\nu} T_{\mu \nu}^{(A)}=0$ but it is not transverse in the index $\mu$, it can in principle contain additional terms that are proportional to the tensors $q^{\mu} \tilde{t}^{\nu}, q^{\mu} \tilde{b}^{\nu}, q^{\mu} \tilde{u}^{\nu}$. However, an inspection of Eq. (3.17) reveals that $T_{\mu \nu}^{(A)}$ is a true tensor rather than a pseudo-tensor and therefore the term $q^{\mu} \tilde{t}^{\nu}$ cannot be present, and in addition that there is no term proportional to $b_{\mu} b_{\nu}$, which is a consequence of the calculation being up to linear terms in $B$. Consequently $T_{\mu \nu}^{(A)}$ is of the form

$$
T_{\mu \nu}^{(A)}=T_{L}^{(A)} Q_{\mu \nu}+T_{T}^{(A)}\left(\tilde{g}_{\mu \nu}-Q_{\mu \nu}\right)+T_{A}^{(A)} A_{3 \mu \nu}+T_{S}^{(A)} S_{3 \mu \nu}+T_{u}^{(A)} q_{\mu} \tilde{u}_{\nu}+T_{b}^{(A)} q_{\mu} \tilde{b}_{\nu},
$$

where the integral formulas for the form factors are obtained by projecting Eq. (3.17) with the tensors that appear in this expansion. In this way, the form factors are given by

$$
\begin{array}{ll}
T_{X}^{(A)}=4|e| B C_{X} \int \frac{d^{4} p}{(2 \pi)^{3}} \eta_{e}(p)\left\{\frac{-K_{X}^{(1)} \delta^{\prime}\left(p^{2}-m^{2}\right)}{(p+q)^{2}-m^{2}}+\frac{K_{X}^{(2)} \delta\left(p^{2}-m^{2}\right)}{\left[(p+q)^{2}-m^{2}\right]^{2}}+(q \rightarrow-q)\right\} & (X=L, T, S, A), \\
T_{X}^{(A)}=4|e| B C_{X} \int \frac{d^{4} p}{(2 \pi)^{3}} \eta_{e}(p)\left\{\frac{-K_{X}^{(1)} \delta^{\prime}\left(p^{2}-m^{2}\right)}{(p+q)^{2}-m^{2}}+\frac{K_{X}^{(2)} \delta\left(p^{2}-m^{2}\right)}{\left[(p+q)^{2}-m^{2}\right]^{2}}-(q \rightarrow-q)\right\} & (X=u, b),
\end{array}
$$

where

$$
\begin{aligned}
C_{A}=C_{L}=2 C_{T} & =\frac{1}{\tilde{u}^{2}}, \\
C_{S} & =\left(\frac{1}{\tilde{u}^{2} \tilde{b}^{2}}\right), \\
C_{u} & =\left(\frac{1}{q^{2} \tilde{u}^{2}}\right), \\
C_{b} & =\left(\frac{1}{q^{2} \tilde{b}^{2}}\right),
\end{aligned}
$$


and

$$
\begin{aligned}
K_{L}^{(1)} & =2 p_{\tilde{u}} K^{\prime}-\tilde{u}^{2} K, \\
K_{L}^{(2)} & =2 p_{\tilde{u}}\left(K^{\prime}-q_{b}\right)+\tilde{u}^{2} K, \\
K_{T}^{(1)} & =-2 p_{\tilde{u}} K^{\prime}-\tilde{u}^{2} K, \\
K_{T}^{(2)} & =-2 p_{\tilde{u}}\left(K^{\prime}-q_{b}\right)+\tilde{u}^{2} K, \\
K_{S}^{(1)} & =p_{\tilde{b}} K^{\prime}+p_{\tilde{u}} p_{u} \tilde{b} \cdot b, \\
K_{S}^{(2)} & =p_{\tilde{b}}\left(K^{\prime}-q_{b}\right)+p_{\tilde{u}}\left(p_{u}+q_{u}\right) \tilde{b} \cdot b, \\
K_{u}^{(1)} & =\left(p \cdot q+q^{2}\right) K^{\prime}+p_{\tilde{u}} K+m^{2} q_{b} \\
K_{u}^{(2)} & =p \cdot q\left(K^{\prime}-q_{b}\right)+p_{\tilde{u}} K+m^{2} q_{b}, \\
K_{b}^{(1)} & =p_{\tilde{b}} K+\tilde{b} \cdot b\left[p_{u}\left(p \cdot q+q^{2}\right)-m^{2} q_{u}\right], \\
K_{b}^{(2)} & =p_{\tilde{b}} K+\tilde{b} \cdot b\left[\left(p_{u}+q_{u}\right) p \cdot q-m^{2} q_{u}\right], \\
K_{A}^{(1)}=K_{A}^{(2)} & =-m^{2},
\end{aligned}
$$

where $K$ is defined in Eq. (4.19),

$$
K^{\prime}=\tilde{u} \cdot b p_{u}-\tilde{u}^{2} p_{b},
$$

and, in addition to Eq. (4.20), we have used the shorthand notation

$$
\begin{aligned}
p_{\tilde{b}} & =p \cdot \tilde{b}, \\
p_{\tilde{u}} & =p \cdot \tilde{u} .
\end{aligned}
$$

In writing Eq. (4.24) we have used the fact that $\tilde{u}^{\mu}$ and $\tilde{b}^{\mu}$ are orthogonal to $q^{\mu}$, as well as the relations $u \cdot \tilde{b}=0$ and $\tilde{u}^{2} \tilde{b} \cdot b=\tilde{b}^{2}$.

\section{Generic formulas}

The integrals involved to evaluate the form factors are of the generic form

$$
\begin{aligned}
& I_{1}=(-1) \int \frac{d^{4} p}{(2 \pi)^{3}} \eta_{e}(p) \frac{F_{1}(p, q) \delta^{\prime}\left(p^{2}-m^{2}\right)}{(p+q)^{2}-m^{2}}, \\
& I_{2}=\int \frac{d^{4} p}{(2 \pi)^{3}} \eta_{e}(p) \frac{F_{2}(p, q) \delta\left(p^{2}-m^{2}\right)}{\left[(p+q)^{2}-m^{2}\right]^{2}} .
\end{aligned}
$$

The various form factors differ only in the choice of the functions $F_{1,2}$. In $I_{2}$, the integration over $p^{0}$ can be carried out trivially, and

$$
I_{2}=\left.\int \frac{d^{3} p}{(2 \pi)^{3} 2 E}\left[\frac{F_{2}(p, q) f_{e}}{\left[q^{2}+2 p \cdot q\right]^{2}}+\frac{F_{2}(-p, q) f_{\bar{e}}}{\left[q^{2}-2 p \cdot q\right]^{2}}\right]\right|_{p^{0}=E(|\vec{p}|)}
$$

where

$$
E(p)=\sqrt{p^{2}-m^{2}} .
$$

Using the relation

$$
\partial_{\mu} \delta\left(p^{2}-m^{2}\right)=2 p_{\mu} \delta^{\prime}\left(p^{2}-m^{2}\right),
$$

the variable $p^{0}$ can also be integrated out in $I_{1}$ by writing

$$
\delta^{\prime}\left(p^{2}-m^{2}\right)=\frac{1}{2 p \cdot u} u^{\mu} \partial_{\mu} \delta\left(p^{2}-m^{2}\right),
$$


followed by an integration by parts. Thus,

$$
\begin{aligned}
I_{1} & =u^{\mu} \int \frac{d^{4} p}{(2 \pi)^{3}} \delta\left(p^{2}-m^{2}\right) \partial_{\mu}\left[\left(\frac{1}{2 p \cdot u}\right) \frac{\eta_{e} F_{1}(p, q)}{(p+q)^{2}-m^{2}}\right] \\
& =\left.u^{\mu} \int \frac{d^{3} p}{(2 \pi)^{3} 2 E}\left\{\partial_{\mu}\left[\left(\frac{1}{2 p \cdot u}\right)\left(\frac{F_{1}(p, q) f_{e}}{(p+q)^{2}-m^{2}}+\frac{F_{1}(-p, q) f_{\bar{e}}}{(p-q)^{2}-m^{2}}\right)\right]\right\}\right|_{p^{0}=E(|\vec{p}|)} .
\end{aligned}
$$

In this fashion, we can write down the integral formulas for all the form factors in terms of a pair of three-dimensional momentum integrals of the form of $I_{1,2}$, with the appropriate choices of the functions $F_{1,2}$ that can be read-off directly from Eqs. (4.17) and (4.22). There are no further manipulations that can be made to the integrals $I_{1,2}$ that will allow us to evaluate the form factors in a general way. However, as we show below, the above formulas are a useful starting point to compute explicitly the form factors in various limiting cases.

\section{APPROXIMATE FORMULAS}

\section{A. Long wavelength limit}

A useful approximation, which is valid in many practical situations, results from taking the so-called long wavelength limit. The approximation is valid in the regime in which the photon momentum $q \ll\langle\mathcal{E}\rangle$, where $\langle\mathcal{E}\rangle$ denotes a typical average energy of the particles in the background. The method to obtain the approximate formulas in this limit is the same that was used in Ref. [3] and we will therefore omit here some of the details.

For the remainder of this section we set $u^{\mu}=(1, \overrightarrow{0})$, so that all the kinematical variables refer to the medium rest frame as usual. Let us consider first $I_{2}$. The method involves making the substitutions $\vec{p} \rightarrow \vec{p} \mp \frac{1}{2} \vec{Q}$ in the two terms in the integrand of Eq. (4.28), respectively, and then making a Taylor expansion in powers of $q / E$. A useful auxiliary formula that is obtained as we have described is

$$
\int \frac{d^{3} p}{(2 \pi)^{3}} \frac{\mathcal{F}(p)}{\left[q^{2}+\lambda 2 p \cdot q\right]^{n}}=\lambda^{n} \int \frac{d^{3} p}{(2 \pi)^{3}} \frac{\left(\mathcal{F}-\lambda \frac{\vec{Q}}{2} \cdot \frac{d \mathcal{F}}{d \vec{p}}-\lambda \frac{\omega}{E} \mathcal{F}\right)\left(1-\frac{\lambda n \omega}{E}\right)}{[2 E \omega-2 \vec{p} \cdot \vec{Q})]^{n}},
$$

where $p^{0}=E(|\vec{p}|)$ and $\lambda= \pm 1$, and the total derivative on the right-hand side is defined as

$$
\frac{d}{d \vec{p}}=\frac{\partial}{\partial \vec{p}}+\frac{\vec{p}}{E} \frac{\partial}{\partial E}
$$

In order to write the resulting formula for $I_{2}$ concisely, it is useful to define

$$
F_{2}^{ \pm}(p, q)=\left.\left[\frac{f_{e}\left(p^{0}\right) F_{2}(p, q) \pm f_{\bar{e}}\left(p^{0}\right) F_{2}(-p, q)}{2 p^{0}}\right]\right|_{p^{0}=E(|\vec{p}|)}
$$

Then using Eq. (5.1), the procedure that we have outlined yields

$$
I_{2}=\int \frac{d^{3} p}{(2 \pi)^{3}} \frac{F_{2}^{+}-\frac{\vec{Q}}{2} \cdot \frac{d F_{2}^{-}}{d \vec{p}}-\frac{\omega}{E} F_{2}^{-}}{[2 E(\omega-\vec{v} \cdot \vec{Q})]^{2}},
$$

where

$$
\vec{v}=\frac{\vec{p}}{E}
$$

The result for $I_{1}$ is slightly more involved algebraically, but straightforward to derive also. In analogy with Eq. (5.3) it is useful to define

$$
\begin{aligned}
F_{1}^{ \pm}(p, q) & =\left.\left[\frac{f_{e}\left(p^{0}\right) F_{1}(p, q) \pm f_{\bar{e}}\left(p^{0}\right) F_{1}(-p, q)}{2 p^{0}}\right]\right|_{p^{0}=E(p)}, \\
F_{1}^{\prime \pm}(p, q) & =\frac{1}{2 E} \frac{\partial F_{1}^{ \pm}(p, q)}{\partial E}
\end{aligned}
$$


in terms of which the long wave limit formula can be expressed in the form

$$
I_{1}=\int \frac{d^{3} p}{(2 \pi)^{3}}\left\{\frac{F_{1}^{\prime-}-\frac{\vec{Q}}{2} \cdot \frac{d F_{1}^{\prime+}}{d \vec{p}}-\frac{\omega}{2 E} F_{1}^{\prime+}}{2 E(\omega-\vec{v} \cdot \vec{Q})}-\frac{F_{1}^{+}-\frac{\vec{Q}}{2} \cdot \frac{d F_{1}^{-}}{d \vec{p}}}{[2 E(\omega-\vec{v} \cdot \vec{Q})]^{2}}\right\} .
$$

Since we are concerned only with the real part of the form factors, the singularity of the integrand is to be handled by taking the principal value part of the integral, as usual in these circumstances.

The formulas in Eqs. (5.4) and (5.7) are particularly useful for computing explicitly the form factors in various limiting cases, as we now illustrate.

\section{B. Static limit and the neutrino induced charge}

Similarly to the situation in the absence of the magnetic field [1], it is possible to some extent to interpret some of the form factors, or certain combinations of them, in terms of electromagnetic moments of the neutrino. In order to identify the static electromagnetic moments we have to look at the vertex function in the static limit $\omega \rightarrow 0$ first, and then take the long wavelength limit $Q \rightarrow 0$. It is well known that taking the limit in the reverse order is not equivalent, since it represents a different physical condition [23].

However, in the present case there is the additional complication that the vertex function, and whence the form factors, are not isotropic functions of $\vec{Q}$ and instead they depend on the components $Q_{\|}$and $Q_{\perp}$ separately. Therefore, the $Q \rightarrow 0$ limit can be taken in two different ways, and in general the form factors will have different corresponding limit value. In order to keep this clear, we use the notation $\Gamma_{\mu}\left(\omega, Q_{\perp}, Q_{\|}\right)$to denote the vertex function for any value of $q$, and $\Gamma_{\mu}\left(0,0, Q_{\|} \rightarrow 0\right)$ or $\Gamma_{\mu}\left(0, Q_{\perp} \rightarrow 0,0\right)$ for its value in the two limits that we have indicated. We use a similar notation for the form factors.

If we insist in identifying a neutrino induced charge, or some other induced electromagnetic moment for that matter, we are thus forced to define two different quantities, corresponding to the two different limits. For example, the definition of the neutrino induced charge used in Ref. 24] for the case of an isotropic medium, must be amended as follows

$$
\begin{aligned}
& e_{\nu_{\ell}}^{\|}=\frac{1}{2 E_{\nu}} \operatorname{Tr} L \not k \Gamma_{0}^{(\ell)}\left(0,0, Q_{\|} \rightarrow 0\right), \\
& e_{\nu_{\ell}}^{\perp}=\frac{1}{2 E_{\nu}} \operatorname{Tr} L \not k \Gamma_{0}^{(\ell)}\left(0, Q_{\perp} \rightarrow 0,0\right),
\end{aligned}
$$

where $k^{\mu}=\left(E_{\nu}, \vec{k}\right)$ is the neutrino momentum vector. For illustrative purposes we consider the evaluation specifically of the form factors that enter in these two formulas.

We consider $e_{\nu_{\ell}}^{\|}$first, which corresponds to set $\vec{Q}_{\perp}=0$, and then take the limit as $Q_{\|} \rightarrow 0$ last. As a practical trick, this amounts to evaluating the vertex function for

$$
q^{\mu}=Q_{\|} b^{\mu},
$$

and then take the $Q_{\|} \rightarrow 0$ limit. We therefore look at

$$
\Gamma_{0}^{(\ell)}\left(0,0, Q_{\|}\right)=\left(\frac{-e g^{2} \chi_{A}^{(\ell)}}{4 M_{W}^{2}}\right)\left\{T_{L}^{(A)} \gamma_{0}-Q_{\|} T_{u}^{(A)} \vec{\gamma} \cdot \hat{b}\right\} L,
$$

where the form factors are evaluated at $\omega=0$ and $Q_{\perp}=0$. From the above formula for $e_{\nu_{\ell}}^{\|}$, Eq. (5.10) represents a contribution to that quantity given by

$$
e_{\nu_{\ell}}^{\|}=\left.\left(\frac{-e g^{2} \chi_{A}^{(\ell)}}{4 M_{W}^{2}}\right) \lim _{Q_{\|} \rightarrow 0}\left[T_{L}^{(A)}-Q_{\|} T_{u}^{(A)} \hat{k} \cdot \hat{b}\right]\right|_{\omega=0, Q_{\perp}=0}
$$

where $\hat{k}$ is the neutrino momentum unit vector.

In order to evaluate $T_{L}^{(A)}$ and $T_{u}^{(A)}$ in this limit we proceed as follows. First, from Eq. (4.24), for $q^{\mu}=Q_{\|} b^{\mu}$,

$$
\begin{aligned}
& K_{L}^{(2)}=K_{L}^{(1)}=2 p^{0} P_{\|}+p^{0} Q_{\|}, \\
& K_{u}^{(2)}=K_{u}^{(1)}=\left(-Q_{\|}\right)\left[p^{02}+P_{\|}^{2}+m^{2}+Q_{\|} P_{\|}\right],
\end{aligned}
$$


where we have decomposed

$$
\vec{p}=\vec{P}_{\perp}+P_{\|} \hat{b} .
$$

For the particular kinematic configuration that we are considering, the integrands do not depend explicitly on $\vec{P}_{\perp}$, but only implicitly through $E(|\vec{p}|)$. Therefore, the derivatives that appear in Eq. (5.6) can be rewritten using

$$
\frac{1}{2 E} \frac{\partial}{\partial E}=\frac{\partial}{\partial P_{\perp}^{2}} .
$$

Applying the results given in Eqs. (5.4) and (5.7), the integral formula for $T_{L}^{(A)}$ becomes

$$
\begin{aligned}
T_{L}^{(A)}\left(0,0, Q_{\|}\right) & =(4|e| B) \int \frac{d^{2} \vec{P}_{\perp} d P_{\|}}{(2 \pi)^{3}}\left(\frac{-1}{2 P_{\|}}\right) \frac{\partial}{\partial P_{\perp}^{2}}\left[\left(1-P_{\|} \frac{\partial}{\partial P_{\|}}\right)\left(f_{e}+f_{\bar{e}}\right)\right] \\
& =0
\end{aligned}
$$

by symmetric integration over $P_{\|}$. Similarly,

$$
T_{u}^{(A)}\left(0,0, Q_{\|}\right)=\frac{-1}{Q_{\|}}(4|e| B) \int \frac{d^{2} \vec{P}_{\perp} d P_{\|}}{(2 \pi)^{3}} \frac{\partial}{\partial P_{\perp}^{2}}\left[\left(\frac{1}{2 E^{2}}-\frac{1}{2 P_{\|}} \frac{\partial}{\partial P_{\|}}\right) E\left(f_{e}+f_{\bar{e}}\right)\right] .
$$

Putting $d^{2} \vec{P}_{\perp}=\pi d P_{\perp}^{2}$ the integral over the transverse components can be performed trivially, and only the contribution from $P_{\perp}^{2}=0$ survives. The remaining integral over $P_{\|}$can be written in a simple form yielding

$$
T_{u}^{(A)}\left(0,0, Q_{\|}\right)=\frac{(4|e| B)}{Q_{\|}} t_{\|}
$$

where

$$
t_{\|}=-\left.\frac{1}{2} \int_{0}^{\infty} \frac{d p}{(2 \pi)^{2}}\left[\frac{\partial}{\partial E}\left(f_{e}(E)+f_{\bar{e}}(E)\right)\right]\right|_{E=E(p)} .
$$

Using Eqs. (5.15) and (5.17) in Eq. (5.11), we finally obtain

$$
e_{\nu_{\ell}}^{\|}=\left(\frac{-e^{2} g^{2} \chi_{A}^{(\ell)}}{M_{W}^{2}}\right)(\hat{k} \cdot \vec{B}) t_{\|} .
$$

The integral $t_{\|}$cannot be evaluated in closed form for an arbitrary distribution, but for particular cases it is readily evaluated.

\section{Non-relativistic classical gas}

For example, in the classical and non-relativistic limit we use

$$
\frac{\partial f_{e, \bar{e}}}{\partial E}=-\beta f_{e, \bar{e}}
$$

where $\beta$ is the inverse temperature, and obtain

$$
\begin{aligned}
& t_{\|}=\frac{\left(n_{e}+n_{\bar{e}}\right) \beta^{2}}{8 m_{e}} . \\
& \text { 2. Degenerate gas }
\end{aligned}
$$

In this case, neglecting $f_{\bar{e}}$,

$$
t_{\|}=\left(\frac{E_{F}}{8 \pi^{2} p_{F}}\right)
$$


where $p_{F}=\left(3 \pi^{2} n_{e}\right)^{1 / 3}$ is the Fermi momentum of the electron gas and $E_{F}$ the corresponding energy. This result holds whether the gas is relativistic or not.

A formula that resembles Eq. (5.19) was obtained in Ref. 21. Although it was not explicitly stated there, upon close inspection it becomes clear that the authors of that reference took the $\vec{Q} \rightarrow 0$ limit by setting $Q_{\perp}=0$ first, and then letting $Q_{\|} \rightarrow 0$ afterwards. Whence their calculation corresponds to the calculation of the quantity that we have identified as $e_{\nu}^{\|}$. However, our result given in Eq. (5.19) differs from the formula that is inferred from the results obtained in Ref. 21] [Eqs. (60) and (61)]. In fact, we can reproduce the result of that reference if we use the relation given in Eq. (5.20) to eliminate the derivatives of the distribution functions in Eq. (5.18). However, Eq. (5.20) is not valid for a general Fermi-Dirac distribution, but only for the case of a non-relativistic and classical gas. For other cases, the formula for the induced charge obtained in that reference cannot be used. The appropriate formula for a degenerate gas is obtained by using Eq. (5.22) or, for an arbitrary distribution, by using Eq. (5.18).

A particular feature of Eq. (5.19), which was first noted in Ref. 21], is the term $\hat{k} \cdot \vec{B}$, which indicates a dependence of $e_{\nu}^{\|}$on the direction of propagation of the neutrino. It is important to keep in mind that this is a kinematic factor that arises from taking the matrix element of the vertex function between the neutrino spinors. The observation that we have made above regarding the non-uniqueness of the zero momentum limit refers to the dependence of the form factors themselves on the variables $Q_{\perp}$ and $Q_{\|}$, independently of such kinematic terms that may enter in specific matrix elements.

For completeness, we summarize below the results of the calculation of $e_{\nu_{\ell}}^{\perp}$ which we have carried out following a similar procedure.

\section{The induced charge $e_{\nu_{\ell}}^{\perp}$}

For $\omega=0$ and $Q_{\|}=0$, we have

$$
\begin{aligned}
T_{\mu 0}^{(V)} & =\frac{1}{Q_{\perp}^{2}} T_{2}^{(V)} i \epsilon_{\mu \alpha \beta \gamma} u^{\alpha} b^{\beta} q^{\gamma}, \\
T_{\mu 0}^{(A)} & =\left(T_{S}^{(A)}-T_{A}^{(A)}\right) b_{\mu}+T_{A}^{(L)} u_{\mu}+T_{u}^{(A)} q_{\mu} .
\end{aligned}
$$

Either from the complete formulas given Eq. (4.22), or from their long wavelength limit versions, it is easy to deduce that

$$
T_{L}^{(A)}\left(0, Q_{\perp}, 0\right)=T_{u}^{(A)}\left(0, Q_{\perp}, 0\right)=0,
$$

by focusing on the integration over $P_{\|}$and observing that in both cases the integrand is an odd function of that variable in this configuration. A less trivial result, but which follows straightforwardly by using the formulas given in Eqs. (5.4) and (5.7) is

$$
T_{2}^{(V)}\left(0, Q_{\perp} \rightarrow 0,0\right)=Q_{\perp}^{2} \times \text { const }
$$

Therefore,

$$
\Gamma_{0}^{(\ell)}\left(0, Q_{\perp} \rightarrow 0,0\right)=\left.\left(\frac{-e g^{2} \chi_{A}^{(\ell)}}{4 M_{W}^{2}}\right) \lim _{Q_{\perp} \rightarrow 0}\left[\left(T_{S}^{A)}-T_{A}^{(A)}\right) \not b L\right]\right|_{\omega=0, Q_{\|}=0} .
$$

Applying Eqs. (5.4) and (5.7) once again to evaluate the combination of form factors $T_{S}^{A)}-T_{A}^{(A)}$ we find

$$
T_{S}^{(A)}\left(0, Q_{\perp} \rightarrow 0,0\right)-T_{A}^{(A)}\left(0, Q_{\perp} \rightarrow 0,0\right)=4|e| B t_{\perp},
$$

where

$$
t_{\perp}=\left.\int \frac{d^{3} p}{(2 \pi)^{3}}\left[\frac{1}{2 E} \frac{\partial}{\partial E}\left\{\frac{1}{2 E} \frac{\partial}{\partial E}\left(\frac{\left(E^{2}+P_{\|}^{2}+m^{2}\right)\left(f_{e}+f_{\bar{e}}\right)}{2 E}\right)\right\}\right]\right|_{E=E(|\vec{p}|)}
$$

and therefore, from Eq. (5.8),

$$
e_{\nu_{\ell}}^{\perp}=\left(\frac{-e^{2} g^{2} \chi_{A}^{\ell}}{M_{W}^{2}}\right)(\hat{k} \cdot \vec{B}) t_{\perp}
$$


For the case of a non-relativistic electron gas, Eq. (5.28) can be manipulated to yield

$$
t_{\perp}=-\left.\frac{1}{2} \int_{0}^{\infty} \frac{d p}{(2 \pi)^{2}}\left[\frac{\partial}{\partial E}\left(f_{e}(E)+f_{\bar{e}}(E)\right)\right]\right|_{E=E(p)} \quad \text { (non-relativistic case) }
$$

which is exactly the same as the integral in Eq. (5.18). Therefore in this limit,

$$
e_{\nu_{\ell}}^{\perp}=e_{\nu_{\ell}}^{\|} \quad(\text { non-relativistic case) } .
$$

The result given in Eq. (5.30), and consequently the equality between $e_{\nu_{\ell}}^{\|}$and $e_{\nu_{\ell}}^{\|}$, is valid whether the gas is classical or degenerate, or in fact for any Fermi-Dirac distribution consistent with the non-relativistic limit. For a relativistic gas, the integrals $t_{\perp}$ and $t_{\|}$are different, and Eq. (5.31) no longer holds.

\section{CONCLUSIONS}

In this work we have been concerned with the electromagnetic properties of a neutrino that propagates in a magnetized electron background. Our goal has been to determine the neutrino electromagnetic vertex function systematically, and in a way that it be useful to study the neutrino processes that may occur in such media, such as Cherenkov radiation and plasmon decay.

Our starting point was to use the electron propagator in the presence of a magnetic field to obtain the oneloop formula for the vertex function up to linear terms in the magnetic field. We then decomposed the vertex function in terms of the minimal and complete set of tensors, consistent with basic physical requirements such as the transversality condition, and obtained the expressions for the form factors in terms of a set of integrals over the momentum distribution functions of the background electrons. Simpler approximate formulas that are valid in the long wavelength limit, and which are useful for practical calculations of the form factors, were given.

For illustrative purposes, and to make contact with previous work, the calculation of the form factors that enter in the effective neutrino charge was considered in some detail. In connection with this we pointed out that, in contrast with the situation in an isotropic medium (i.e., in the absence of the magnetic field), the static form factors do not have a unique value in the zero (photon) momentum limit. We stress once more that this is not a mathematical ambiguity, but it is actually a physical effect that results from the fact that the medium in the present case is essentially anisotropic. Furthermore, it is dynamical effect quite different from the kinematic dependence that the matrix elements of the vertex function may have on the direction of propagation of the neutrino. Thus, for the specific case of the neutrino induced charge, the two quantities $e_{\nu}^{\|}$and $e_{\nu}^{\perp}$ were introduced and their expressions in terms of the form factors were given. As we showed, they have the same value for a non-relativistic gas, but are given by different formulas otherwise. In a given application all the form factors are in principle relevant. The same method that we have used to evaluate those that are related to the neutrino induced charge, can be used similarly to yield the corresponding formulas for all the others.

In this work we have taken into account only the electrons in the background. While the effects of the nucleons are sometimes suppressed due to their mass, it is known that in the presence of a magnetic field their contribution to some of the form factors is important. The method and formulas that we have presented here provide a firm basis, both conceptual and practical, to take those effects into account as well. They provide a consistent basis to continue and extend the investigation of problems in which the electromagnetic properties of the neutrino and its coupling to a magnetic field are believed to be important.

\section{Acknowledgments}

This material is based upon work supported by the US National Science Foundation under Grant No. 0139538.

\section{APPENDIX A: PROOF OF THE TRANSVERSALITY RELATIONS FOR $T_{\mu \nu}^{(V)}$}

We consider in some detail the proof of the relation

$$
q^{\nu} T_{\mu \nu}^{(V)}=0
$$

where $T_{\mu \nu}^{(V)}$ is given in Eq. (3.17). Since $T_{\mu \nu}^{(V)}$ is antisymmetric, this implies that $q^{\mu} T_{\mu \nu}^{(V)}=0$ also. 
We write $T_{\mu \nu}^{(V)}$ in the following form

$$
\left.T_{\mu \nu}^{(V)}=(4|e| B) \int \frac{d^{4} p}{(2 \pi)^{3}} \eta_{e}(p) I_{\mu \nu}^{(T B} B\right)
$$

where

$$
I_{\mu \nu}^{(T B)} \equiv\left\{\frac{-L_{\mu \nu}^{(1)} \delta^{\prime}\left(p^{2}-m^{2}\right)}{d}+\frac{L_{\mu \nu}^{(2)} \delta\left(p^{2}-m^{2}\right)}{d^{2}}\right\}-(q \rightarrow-q) .
$$

and

$$
d \equiv p^{\prime 2}-m^{2} .
$$

Let us define the quantity

$$
I_{\mu \nu}^{(T)} \equiv \delta\left(p^{2}-m^{2}\right)\left[\frac{L_{\mu \nu}}{d}+(q \rightarrow-q)\right],
$$

where

$$
L_{\mu \nu}=2 p_{\mu} p_{\nu}+p_{\mu} q \nu+q_{\mu} p_{\nu}-(p \cdot q) g_{\mu \nu} .
$$

We now state the following result, which is a purely algebraic identity. Defining

$$
P^{\mu \nu} \equiv i \epsilon^{\mu \nu \alpha \beta} b_{\alpha} u_{\beta},
$$

then

$$
q^{\mu} I_{\mu \nu}^{(T B)}=\frac{i}{2} P^{\lambda \mu} \partial_{\lambda} I_{\mu \nu}^{(T)},
$$

where $\partial_{\lambda} \equiv \partial / \partial p^{\lambda}$. We prove Eq. (A8) below but, for the moment, notice that it is all that we need. Observing that

$$
P^{\lambda \mu} \partial_{\lambda} \eta(p)=0
$$

(because $\partial_{\lambda} \eta \propto u_{\lambda}$ ), then from Eqs. (A2) and w8 have

$$
\begin{aligned}
q^{\mu} T_{\mu \nu}^{(V)} & =(4|e| B) \frac{i}{2} P^{\lambda \mu} \int \frac{d^{4} p}{(2 \pi)^{3}} \partial_{\lambda}\left[\eta_{e}(p) I_{\mu \nu}^{(T)}\right] \\
& =0 .
\end{aligned}
$$

\section{Proof of Eq. (A8)}

To prove Eq. (A8), let us consider first its right-hand side,

$$
P^{\lambda \mu} \partial_{\lambda} I_{\mu \nu}^{(T)}=P^{\lambda \mu}\left\{\partial_{\lambda}\left(\frac{\delta\left(p^{2}-m^{2}\right) L_{\mu \nu}}{d}\right)+(q \rightarrow-q)\right\}
$$

Using

$$
\begin{aligned}
\partial_{\lambda} \delta\left(p^{2}-m^{2}\right) & =\delta^{\prime}\left(p^{2}-m^{2}\right)\left(2 p_{\lambda}\right) \\
\partial_{\lambda}\left(\frac{1}{d}\right) & =\frac{-2}{d^{2}} p_{\lambda}^{\prime} \\
\partial_{\lambda} L_{\mu \nu} & =2 g_{\mu \lambda} p_{\nu}+2 g_{\nu \lambda} p_{\mu}+g_{\mu \lambda} q_{\nu}+g_{\nu \lambda} q_{\mu}-g_{\mu \nu} q_{\lambda},
\end{aligned}
$$

we obtain

$$
\begin{aligned}
P^{\lambda \mu} \partial_{\lambda} I_{\mu \nu}^{(T)}= & \frac{2 i \delta^{\prime}\left(p^{2}-m^{2}\right)}{d}\left[p_{\nu} \epsilon^{\mu \lambda \alpha \beta} q_{\mu} p_{\lambda} u_{\alpha} b_{\beta}-(p \cdot q) \epsilon_{\nu \lambda \alpha \beta} p^{\lambda} u^{\alpha} b^{\beta}\right] \\
& -\frac{2 i \delta\left(p^{2}-m^{2}\right)}{d^{2}}\left[p_{\nu}^{\prime} \epsilon^{\mu \lambda \alpha \beta} p_{\mu} q_{\lambda} u_{\alpha} b_{\beta}-(p \cdot q) \epsilon_{\nu \lambda \alpha \beta} p^{\prime \lambda} u^{\alpha} b^{\beta}\right] \\
& -\frac{2 i \delta\left(p^{2}-m^{2}\right)}{d} \epsilon_{\nu \lambda \alpha \beta} p^{\prime \lambda} u^{\alpha} b^{\beta} \\
& +(q \rightarrow-q) .
\end{aligned}
$$


The third term can be rewritten thus,

$$
\frac{\delta\left(p^{2}-m^{2}\right)}{d}=\frac{\delta\left(p^{2}-m^{2}\right)}{d^{2}}\left(q^{2}+2 p \cdot q\right)
$$

and then combining it with the second term,

$$
\begin{aligned}
P^{\lambda \mu} \partial_{\lambda} I_{\mu \nu}^{(T)}= & \frac{2 i \delta^{\prime}\left(p^{2}-m^{2}\right)}{d}\left[-p_{\nu} \epsilon^{\mu \lambda \alpha \beta} p_{\mu} q_{\lambda} u_{\alpha} b_{\beta}-(p \cdot q) \epsilon_{\nu \lambda \alpha \beta} p^{\lambda} u^{\alpha} b^{\beta}\right] \\
& -\frac{2 i \delta\left(p^{2}-m^{2}\right)}{d^{2}}\left[p_{\nu}^{\prime} \epsilon^{\mu \lambda \alpha \beta} p_{\mu} q_{\lambda} u_{\alpha} b_{\beta}+\left(p^{\prime} \cdot q\right) \epsilon_{\nu \lambda \alpha \beta} p^{\prime \lambda} u^{\alpha} b^{\beta}\right] \\
& +(q \rightarrow-q)
\end{aligned}
$$

This is all we will do with the right-hand side of Eq. (A8).

Now let us consider the left-hand side. From Eq. (3.16), we get

$$
\begin{aligned}
q^{\mu} L_{\mu \nu}^{(1)} & =-(p \cdot u) \epsilon_{\mu \nu \alpha \beta} q^{\mu} p^{\alpha} b^{\beta}+(p \cdot b) \epsilon_{\mu \nu \alpha \beta} q^{\mu} p^{\alpha} u^{\beta}+m^{2} \epsilon_{\mu \nu \alpha \beta} q^{\mu} u^{\alpha} b^{\beta} \\
q^{\mu} L_{\mu \nu}^{(2)} & =\left(p^{\prime} \cdot u\right) \epsilon_{\mu \nu \alpha \beta} q^{\mu} p^{\alpha} b^{\beta}-\left(p^{\prime} \cdot b\right) \epsilon_{\mu \nu \alpha \beta} q^{\mu} p^{\alpha} u^{\beta}-m^{2} \epsilon_{\mu \nu \alpha \beta} q^{\mu} u^{\alpha} b^{\beta}
\end{aligned}
$$

Using the identity given in Eq. 4.10), we obtain the following two identities,

$$
\begin{array}{r}
(p \cdot u) \epsilon_{\mu \nu \alpha \beta} q^{\mu} b^{\alpha} p^{\beta}-(p \cdot b) \epsilon_{\mu \nu \alpha \beta} q^{\mu} u^{\alpha} p^{\beta}+p^{2} \epsilon_{\mu \nu \alpha \beta} q^{\mu} u^{\alpha} b^{\beta}= \\
-p_{\nu} \epsilon_{\mu \lambda \alpha \beta} p^{\mu} q^{\lambda} u^{\alpha} b^{\beta}-(p \cdot q) \epsilon_{\nu \lambda \alpha \beta} p^{\lambda} u^{\alpha} b^{\beta}
\end{array}
$$

and

$$
\begin{array}{r}
\left(p^{\prime} \cdot u\right) \epsilon_{\mu \nu \alpha \beta} q^{\mu} b^{\alpha} p^{\beta}-\left(p^{\prime} \cdot b\right) \epsilon_{\mu \nu \alpha \beta} q^{\mu} u^{\alpha} p^{\beta}+p^{\prime 2} \epsilon_{\mu \nu \alpha \beta} q^{\mu} u^{\alpha} b^{\beta}= \\
-p_{\nu}^{\prime} \epsilon_{\mu \lambda \alpha \beta} p^{\mu} q^{\lambda} u^{\alpha} b^{\beta}-\left(p^{\prime} \cdot q\right) \epsilon_{\nu \lambda \alpha \beta} p^{\lambda} u^{\alpha} b^{\beta}
\end{array}
$$

and using them we can rewrite Eq. (A16) in the form

$$
\begin{aligned}
& q^{\mu} L_{\mu \nu}^{(1)}=-p_{\nu} \epsilon_{\mu \lambda \alpha \beta} p^{\mu} q^{\lambda} u^{\alpha} b^{\beta}-(p \cdot q) \epsilon_{\nu \lambda \alpha \beta} p^{\lambda} u^{\alpha} b^{\beta}-\left(p^{2}-m^{2}\right) \epsilon_{\mu \nu \alpha \beta} q^{\mu} u^{\alpha} b^{\beta} \\
& q^{\mu} L_{\mu \nu}^{(2)}=p_{\nu}^{\prime} \epsilon_{\mu \lambda \alpha \beta} p^{\mu} q^{\lambda} u^{\alpha} b^{\beta}+\left(p^{\prime} \cdot q\right) \epsilon_{\nu \lambda \alpha \beta} p^{\prime \lambda} u^{\alpha} b^{\beta}+\left(p^{\prime 2}-m^{2}\right) \epsilon_{\mu \nu \alpha \beta} q^{\mu} u^{\alpha} b^{\beta}
\end{aligned}
$$

Therefore,

$$
\begin{aligned}
q^{\mu} I_{\mu \nu}^{(T B)}= & \left\{\frac{-q^{\mu} L_{\mu \nu}^{(1)} \delta^{\prime}\left(p^{2}-m^{2}\right)}{d}+\frac{q^{\mu} L_{\mu \nu}^{(2)} \delta\left(p^{2}-m^{2}\right)}{d^{2}}\right\}-(q \rightarrow-q) \\
= & -\frac{\delta^{\prime}\left(p^{2}-m^{2}\right)}{d}\left[-p_{\nu} \epsilon_{\mu \lambda \alpha \beta} p^{\mu} q^{\lambda} u^{\alpha} b^{\beta}-(p \cdot q) \epsilon_{\nu \lambda \alpha \beta} p^{\lambda} u^{\alpha} b^{\beta}-\left(p^{2}-m^{2}\right) \epsilon_{\mu \nu \alpha \beta} q^{\mu} u^{\alpha} b^{\beta}\right] \\
& +\frac{\delta\left(p^{2}-m^{2}\right)}{d^{2}}\left[p_{\nu}^{\prime} \epsilon_{\mu \lambda \alpha \beta} p^{\mu} q^{\lambda} u^{\alpha} b^{\beta}+\left(p^{\prime} \cdot q\right) \epsilon_{\nu \lambda \alpha \beta} p^{\prime \lambda} u^{\alpha} b^{\beta}+\left(p^{\prime 2}-m^{2}\right) \epsilon_{\mu \nu \alpha \beta} q^{\mu} u^{\alpha} b^{\beta}\right] \\
& +(q \rightarrow-q) .
\end{aligned}
$$

The two terms that contain $\left(p^{2}-m^{2}\right)$ and $\left(p^{\prime 2}-m^{2}\right)$ combine to give

$$
\frac{1}{d}\left[\delta^{\prime}\left(p^{2}-m^{2}\right)\left(p^{2}-m^{2}\right)+\delta\left(p^{2}-m^{2}\right)\right]=0
$$

where we have used the relation

$$
x \delta^{\prime}(x)=-\delta(x)
$$

The remaining terms precisely coincide with Eq. (A15), which therefore proves Eq. (A8). 


\section{APPENDIX B: PROOF OF THE TRANSVERSALITY RELATIONS FOR $T_{\mu \nu}^{(A)}$}

The relation

$$
q^{\nu} T_{\mu \nu}^{(A)}=0
$$

is proven similarly. From Eq. 3.17,

$$
q^{\nu} T_{\mu \nu}^{(A)}=(4|e| B) \int \frac{d^{4} p}{(2 \pi)^{3}} \eta_{e}(p)\left\{\frac{-q^{\nu} K_{\mu \nu}^{(1)} \delta^{\prime}\left(p^{2}-m^{2}\right)}{(p+q)^{2}-m^{2}}+\frac{q^{\nu} K_{\mu \nu}^{(2)} \delta\left(p^{2}-m^{2}\right)}{\left[(p+q)^{2}-m^{2}\right]^{2}}-(q \rightarrow-q)\right\},
$$

where, from the defining equation Eq. (3.16), we obtain

$$
\begin{aligned}
& q^{\nu} K_{\mu \nu}^{(1)}=p_{\mu}[-(p \cdot b)(u \cdot q)+(p \cdot u)(b \cdot q)]+u_{\mu}\left[-(p \cdot b)\left(p^{\prime} \cdot q\right)-m^{2} b \cdot q\right]+b_{\mu}\left[(p \cdot u)\left(p^{\prime} \cdot q\right)+m^{2} u \cdot q\right], \\
& q^{\nu} K_{\mu \nu}^{(2)}=p_{\mu}^{\prime}[-(p \cdot b)(u \cdot q)+(p \cdot u)(b \cdot q)]+u_{\mu}\left[-\left(p^{\prime} \cdot b\right)(p \cdot q)-m^{2} b \cdot q\right]+b_{\mu}\left[\left(p^{\prime} \cdot u\right)(p \cdot q)+m^{2} u \cdot q\right] .
\end{aligned}
$$

Then, in analogy with Eq. (A5), we define

$$
J_{\mu \nu}^{(T)}=\delta\left(p^{2}-m^{2}\right) \frac{\epsilon_{\mu \nu \alpha \beta} p^{\alpha} q^{\beta}}{d}
$$

and by straightforward calculation of $\partial_{\lambda} J_{\mu \nu}^{(T)}$ it follows that

$$
\begin{aligned}
\frac{-q^{\nu} K_{\mu \nu}^{(1)}}{d} \delta^{\prime}\left(p^{2}-m^{2}\right)+\frac{q^{\nu} K_{\mu \nu}^{(2)}}{d^{2}} \delta\left(p^{2}-m^{2}\right)= & \left(\frac{-1}{2}\right)\left[\frac{1}{i} P^{\nu \lambda} \partial_{\lambda} J_{\mu \nu}^{(T)}+\frac{\delta\left(p^{2}-m^{2}\right)}{d}\left(2 Y_{\mu}\right)\right] \\
& +\left[X_{\mu}\left(q^{2}+2 p \cdot q\right)+Y_{\mu}\left(p^{2}-m^{2}\right)\right]\left[\frac{\delta\left(p^{2}-m^{2}\right)}{d^{2}}-\frac{\delta^{\prime}\left(p^{2}-m^{2}\right)}{d}\right] \\
= & \left(\frac{i}{2}\right) P^{\nu \lambda} \partial_{\lambda} J_{\mu \nu}^{(T)}-X_{\mu} \delta^{\prime}\left(p^{2}-m^{2}\right) .
\end{aligned}
$$

In Eq. (B5) we have defined

$$
\begin{aligned}
X_{\mu} & =b_{\mu}(u \cdot p)-u_{\mu}(b \cdot p), \\
Y_{\mu} & =u_{\mu}(b \cdot q)-b_{\mu}(u \cdot q),
\end{aligned}
$$

and to arrive at the last equality we have used the relation of Eq. (A22) once more. Thus, using Eq. (B55) in Eq. (B22),

$$
\begin{aligned}
q^{\nu} T_{\mu \nu}^{(A)} & =(4|e| B) \frac{i}{2} P^{\nu \lambda} \int \frac{d^{4} p}{(2 \pi)^{3}} \partial_{\lambda}\left[\eta_{e}(p) J_{\mu \nu}^{(T)}-(q \rightarrow-q)\right] \\
& =0 .
\end{aligned}
$$

On the other hand, by separating the tensors $K_{\mu \nu}^{(1,2)}$ into their symmetric and antisymmetric parts, it is easy to show that

$$
q^{\mu} T_{\mu \nu}^{(A)}=(4|e| B)\left(4 m^{2} Y_{\nu}\right) \int \frac{d^{4} p}{(2 \pi)^{3}} \eta_{e}(p)\left[\frac{\delta\left(p^{2}-m^{2}\right)}{d^{2}}+(q \rightarrow-q)\right]
$$

which explicitly verifies that, as expected, $T_{\mu \nu}^{(A)}$ is not transverse with respect to its first index. In this respect, the tensor $T_{\mu \nu}^{(A)}$, calculated in the presence of the magnetic field as we re doing here, differs from the corresponding quantity calculated without the magnetic field, in which case it is transverse in the first index as well.

[1] J. F. Nieves and P. B. Pal, Phys. Rev. D 40, 1693 (1989).

[2] V. N. Oraevsky, V. B. Semikoz, and Y. A. Smorodinsky, Sov. Phys. JETP Lett. 43, 709 (1986).

[3] J. C.D’Olivo, J. F. Nieves, and P. B. Pal, Phys. Rev. D 40, 3679 (1989). 
[4] R. F. Sawyer, Phys. Rev. D 46, 1180 (1992).

[5] J. C. D’Olivo, J. F. Nieves, and P. B. Pal, Phys. Rev. Lett. 64, 1088 (1990).

[6] V. B. Semikoz and J. W. F. Valle, Nucl. Phys. B 425, 65 (1994).

[7] V. B. Semikoz and J. W. F. Valle, Nucl. Phys. B 485, 585 (1997).

[8] J. C. D'Olivo and J. F. Nieves, Phys. Lett. B 383, 87 (1996).

[9] J. C. D'Olivo, J. F. Nieves, and P. B. Pal, Phys. Lett. B 365, 178 (1996).

[10] A. Kusenko and G. Segré, Phys. Rev. Lett. 77, 4872 (1996).

[11] A. Kusenko and G. Segré, Phys. Lett. B 396, 197 (1997).

[12] A. Kusenko and G. Segré, Phys. Rev. D 59, 061302 (1999).

[13] A. Kusenko and G. Segré, Phys. Rev. Lett. 79, 2751 (1997).

[14] Y. Z. Qian, Phys. Rev. Lett. 79, 2750 (1997).

[15] H. Nunokawa, V. B. Semikoz, A. Y. Smirnov, and J. W. F. Valle, Nucl. Phys. B 501, 17 (1997).

[16] S. Esposito and G. Capone, Z. Phys. C 70, 55 (1996).

[17] A. Erdas, C. W. Kim, and T. H. Lee, Phys. Rev. D 58, 08516 (1998).

[18] P. Elmfors, D. Grasso, and G. Raffelt, Nucl. Phys. B 479, 3 (1996).

[19] J. C. D'Olivo and J. F. Nieves, Phys. Rev. D 56, 5898 (1997).

[20] A. N. Ioannisian and G. G. Raffelt, Phys. Rev. D 55, 7038 (1997).

[21] K. Bhattacharya, A. Ganguly, and S. Konar, Phys. Rev. D 65, 013007 (2001).

[22] J. C. D'Olivo, J. F. Nieves, and S. Sahu, Phys. Rev. D 67, 025018 (2003).

[23] J. F. Nieves and P. B. Pal, Phys. Rev. D 51, 5300 (1995), and references there in.

[24] J. F. Nieves and P. B. Pal, Phys. Rev. D 49, 1398 (1994). 